\title{
Plantain Herb Extracts significantly attenuate the quorum sensing-controlled virulence factors and inhibit biofilm formation in Pseudomonas aeruginosa PAO1
}

\author{
Shan $\mathrm{Li}^{1}$, Jiangning $\mathrm{Yao}^{2}$, Haoming $\mathrm{Li}^{*}$ \\ ${ }^{1,2}$ School of Biosciences and Biopharmaceutics, Guangdong Pharmaceutical University, Guangzhou Higher Education Mega Center, \\ Guangzhou 510006, China
}

\begin{abstract}
Pseudomonas aeruginosa is a Gram-negative organism that can survive under harsh conditions, and it is also an opportunistic pathogen that can produce cell-associated extracellular virulence factors. Several of these virulence factors have been demonstrated to be regulated by quorum sensing (QS). Plantain Herb has been used as antibacterial agents for many centuries in China. In this study, we analyzed Plantain Herb Extracts (PHE) at the concentration of $16 \mu \mathrm{g} / \mathrm{mL}$ (Group A, MIC), $8 \mu \mathrm{g} / \mathrm{mL}$ (Group B, 1/2 MIC) and 4 $\mu \mathrm{g} / \mathrm{mL}$ (Group C, 1/4 MIC) for inhibition of the virulence factors production and biofilm formation in $P$. aeruginosa PAO1. The virulence factors included pyocyanin, rhamnolipids, protease and alginate. PHE showed significant inhibition of virulence factors as compared to the control group without interfering its growth. Thus, PHE might be a potent QS inhibitor and anti-biofilm agent in the treatment of Pseudomonas aeruginosa infections.
\end{abstract}

\section{Introduction}

Pathogenic bacteria are harmful to human health. Bacterial infection has been a major problem on the medical treatment. With the introduction of natural antibiotics such as penicillin, antibiotic derivatives and synthetic antibiotics, they have opened a new era in the treatment of bacterial infections [1]. However, in modern society, with the widely abuse of antibiotics, drug resistance is becoming a severe problem, at the same time, the emergence of multi-resistant bacteria and mutation time of drug resistance also become more and more short, so it is urgent to find new targets of antibacterial agents and pathways to treat diseases.

Quorum Sensing (QS) is the regulation of gene expression in response to cell density. Bacteria could produce some signaling molecules, called autoinducers (AI), that can coordinate the physiological activities as well as the expression of virulence factors in the pathogenesis [2]. QS can be both found in Gram-negative and Gram-positive bacteria. Signaling molecules or AIs used by Gram-negative bacteria are known as $\mathrm{N}$-acyl homoserine lactones (AHLs), while Gram-positive bacteria utilize post-translationally modified oligopeptides as signaling molecules [3].

PAO1 is an opportunistic pathogenic bacterium, which can be easily detected in the skin and lung infections. QS plays a significant role in the regulation of $P$. aeruginosa virulence expression such as biofilm, pyocyanin, elastase, swarming and protease [4]. As far as we know, there are three clear QS systems in $P$. aeruginosa: PQS system, Rhl system and Las system. RhlI/R and LasI/R are both AHL-depended QS system, which can produce C4-HSL and 3-oxo-C12-HSL respectively $[5,6]$.

Plantain Herb is a Traditional Chinese Medicine (TCM), and it has a long medical history. According to Pharmacopoeia of People's Republic of China, it is a dry whole grass of Plantago asiatica L, used in reducing fever, inducing urination, anti-diarrhea, improving eyesight and expelling phlegm [7]. In this work, we aimed to investigate the potential of Plantain Herb as quorum-sensing inhibitors.

\section{Experimental Section}

\subsection{TCM extraction}

Plantain Herb was purchased from local market in Guangdong, China. Dry whole herb was grounded to fine powder, powder $(5 \mathrm{~g})$ was soaking in $100 \mathrm{~mL}$ of $95 \%$ ethanol for $24 \mathrm{~h}$ and sonicated for $30 \mathrm{~min}$, the extract was concentrated with a rotary evaporator and re-dissolved in $90 \%(\mathrm{v} / \mathrm{v})$ ethanol to the final concentration of $1 \mathrm{mg} / \mathrm{mL}$.

\subsection{Measurement of minimum inhibitory concentration (MIC)}

MIC was determined by the broth micro-dilution method (Clinical and Laboratory Standards Institute [CLSI], 2015). Serial two-fold dilutions method was used in this 
assay, $100 \mu \mathrm{L}$ of PHE solution and $100 \mu \mathrm{L}$ of LB broth were mixed well in the first well of a microtiter plate, extra $10 \mu \mathrm{L}$ of PAO1 culture was added and incubated at $37^{\circ} \mathrm{C}$ for $18 \mathrm{~h}$.

\subsection{Bacterial Growth}

The influence of PHE on the PAO1 growth was examined according to former studies [8]. 1\% overnight PAO1 culture, PHE and LB broth $(0.5 \%$ yeast extract, $1 \%$ tryptone, $1 \% \mathrm{NaCl}$ ) were mixed well in Erlenmeyer flasks and incubated at $37^{\circ} \mathrm{C}$ with shaking. The absorbance of culture turbidity was read at $570 \mathrm{~nm}$ for 24 $\mathrm{h}$ at every 2 hours.

\subsection{Effect of PHE on the production of extracellular virulence factors of $P$. aeruginosa}

Rhamnolipids assay was performed as described by Rasamiravaka et al. [9] with modifications. Briefly, 1\% overnight grown PAO1 culture with or without PHE were cultivated in PPGAS broth $\left(20 \mathrm{mM} \mathrm{NH}_{4} \mathrm{Cl}, 20 \mathrm{mM} \mathrm{KCl}\right.$, $1.6 \mathrm{mM} \mathrm{MgSO}_{4}, 0.5 \%$ Glucose, $1.0 \%$ Peptone, $120 \mathrm{mM}$ Tris- $\mathrm{HCl}$ every $1000 \mathrm{~mL}, \mathrm{pH} 7.2$ ) for $24 \mathrm{~h}$. Cell-free supernatant $(200 \mathrm{Ml}, \mathrm{pH} 2.0)$ was extracted with ethyl acetate $(2 \mathrm{~mL})$ twice. Then the organic solvent was dried overnight and re-dissolved in $500 \mu \mathrm{L}$ of $\mathrm{ddH}_{2} \mathrm{O}, 100 \mu \mathrm{L}$ of resulting solution was mixed completely with $800 \mu \mathrm{L}$ of $60 \%(\mathrm{v} / \mathrm{v})$ sulfuric acid and $100 \mu \mathrm{L}$ of $1.7 \%$ orcinol. The absorbance was read at $421 \mathrm{~nm}$.

Treated or untreated PAO1 culture $(2 \mathrm{~mL})$ was extracted with chloroform $(2 \mathrm{~mL})$ and centrifuged at $10000 \mathrm{rpm}$ for $10 \mathrm{~min}$. The chloroform layer was re-extracted with $1 \mathrm{~mL} \mathrm{HCl}(0.2 \mathrm{M})$. After the same centrifugation, the absorbance of $\mathrm{HCl}$ layer was read at $570 \mathrm{~nm}[10]$. The amount of pyocyanin was calculated by multiplying the OD570 by 17.072 .

Treated or untreated PAO1 culture $(1 \mathrm{~mL})$ was centrifuged at $10000 \mathrm{rmp}$ for $10 \mathrm{~min}$. Then sterile-filtered supernatant $(150 \mu \mathrm{L})$ was mixed with $250 \mu \mathrm{L}$ of $2 \%(\mathrm{w} / \mathrm{v})$ azocasein-Tris- $\mathrm{HCl}$ solution and incubated at $4{ }^{\circ} \mathrm{C}$ for 4 hours, $1.2 \mathrm{~mL}$ of $10 \%(\mathrm{w} / \mathrm{v})$ trichloroacetic acid was added to terminate the reaction and centrifuged at 10000 $\mathrm{rmp}$ for $10 \mathrm{~min}$ [11]. The protease activity was determined by recording the optical density at $440 \mathrm{~nm}$.

Treated or untreated PAO1 culture $(1 \mathrm{~mL})$ was mixed well with $3 \mathrm{~mL}$ of $10 \%(\mathrm{w} / \mathrm{v}) \mathrm{CuSO}_{4}$ solution ( $\mathrm{pH} 4.0$ ). After sat at room temperature for $1 \mathrm{~h}$ and centrifuged at $10000 \mathrm{rpm}$ for $10 \mathrm{~min}$, the sediment was dissolved in 100 $\mu \mathrm{L}$ of $\mathrm{NH}_{3} \cdot \mathrm{H}_{2} \mathrm{O}(1 \mathrm{~N})$ and $900 \mu \mathrm{L}$ of $\mathrm{ddH}_{2} \mathrm{O}$. Then, $2 \mathrm{~mL}$ of 1,3-dihydroxynaphthalene solution and $1 \mathrm{~mL}$ of $\mathrm{M}_{2} \mathrm{ICuO}_{2}$ solution were mixed well with above culture. After boiled for $40 \mathrm{~min}, 4 \mathrm{~mL}$ of cold ethyl acetate was used to extract alginate and $20 \%(\mathrm{w} / \mathrm{v}) \mathrm{NaCl}$ was used to remove impurities [12]. The content of alginate was determined by recording the optical density at $570 \mathrm{~nm}$.

\subsection{The semiquantitative of Biofilm}

This assay was performed as conducted by Busetti et al. [13] with some modifications. $1 \%$ of PAO1 culture was inoculated into LB broth (with or without PHE) containing sterilized coverslips $(2 \mathrm{~cm} \times 2 \mathrm{~cm})$ in 6 -well microplate and incubated at $37^{\circ} \mathrm{C}$. Within the seven days, the solution was changed every $24 \mathrm{~h}$, sterilized PBS (PH 7.8) was used to remove planktonic bacteria and bacteria that did not form a biofilm. $2.5 \%$ pentanediol $(2 \mathrm{~mL})$ was used to fix and stained with $1 \mathrm{~mL}$ of $0.5 \%$ crystal violet. After being rinsed 3 times to remove excessive crystal violet (CV) dye, $2 \mathrm{~mL}$ of $95 \%$ ethanol was used to dissolve CV-bound biofilm. The absorbance was read at $570 \mathrm{~nm}$.

\subsection{Statistical Analysis}

All experiments were performed in triplicates and the data were analyzed using one-way ANOVA test by SPSS 17.0 statistical software.

\section{Results}

\subsection{MIC and The influence of PHE on PAO1 growth}

MIC of PHE against PAO1 was $16 \mu \mathrm{g} / \mathrm{mL}$. Growth assay showed that PHE inhibited PAO1 growth at the concentration of $16 \mu \mathrm{g} / \mathrm{mL}$ (Fig. 1), while there was little effect on the growth curve at $8 \mu \mathrm{g} / \mathrm{mL}$ and $4 \mu \mathrm{g} / \mathrm{mL}$.

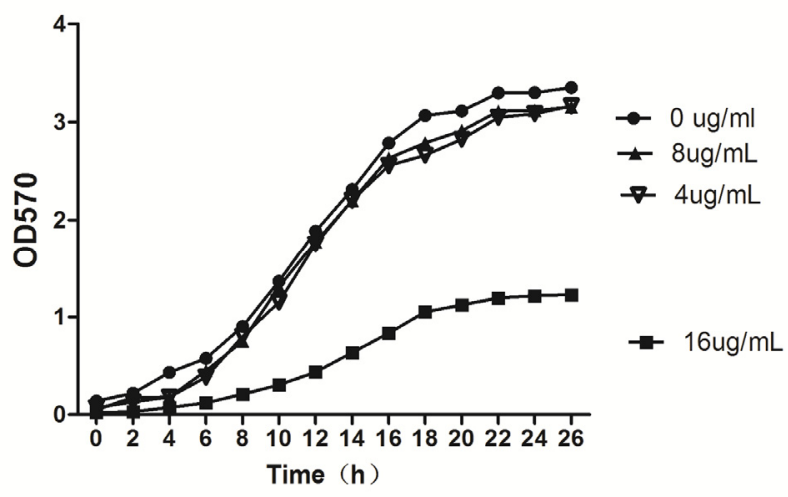

Fig. 1. Growth of $P$. aeruginosa PAO1 in the presence of PHE.

\subsection{PHE decreased the production of extracellular virulence factors of $P$. aeruginosa}

Results showed a significant difference among all positive groups as compared to control $(P<0.01)$. The effect of rhamnolipids inhitation was strongest at 8 $\mu \mathrm{g} / \mathrm{mL}$ of PHE, $60.8 \%$ of decline was obtained. While the group $\mathrm{B}$ and $\mathrm{C}$ decreased rhamnolipids production by $46.8 \%$ and $9.7 \%$ respectively (Fig. $2 \mathrm{a}$ ). 
(a)

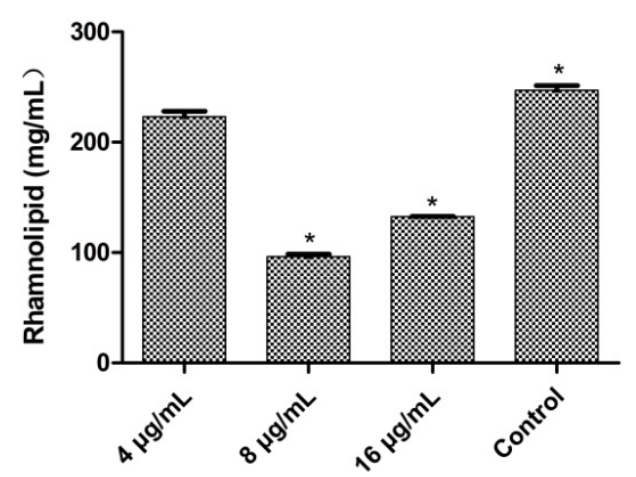

Fig. 2a. The production of Rhamnolipids.

The inhibiting rates of PHE against the production of pyocyanin were $10.2 \%$ (at MIC, $P<0.01$ ), $8.2 \%$ (at $1 / 2$ MIC, $P<0.01$ ) and $7.8 \%$ (at $1 / 4$ MIC) respectively (Fig.2b).

(b)

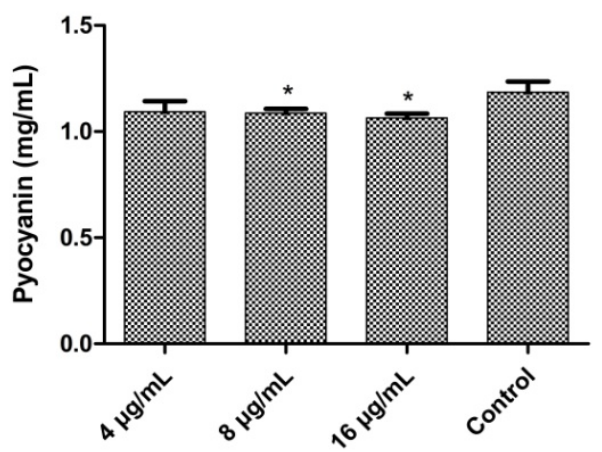

Fig. 2b. Pyocyanin production. *Significance, $P<0.01$.

PHE at $16,8,4 \mu \mathrm{g} / \mathrm{mL}$ reduced protease activity by $37.9 \%, 38.7 \%$ and $14.7 \%$ respectively $(P<0.01$, Fig. 2c).

(c)



Fig. 2c. The protease activity. ${ }^{*}$ Significance, $P<0.01$.

The yield of alginate was significant declined with the interference of PHE. In the present of PHE, almost $96.8 \%, 92.1 \%, 86.4 \%$ inhibition were found in alginate production respectively (Fig. 2 d). (d)

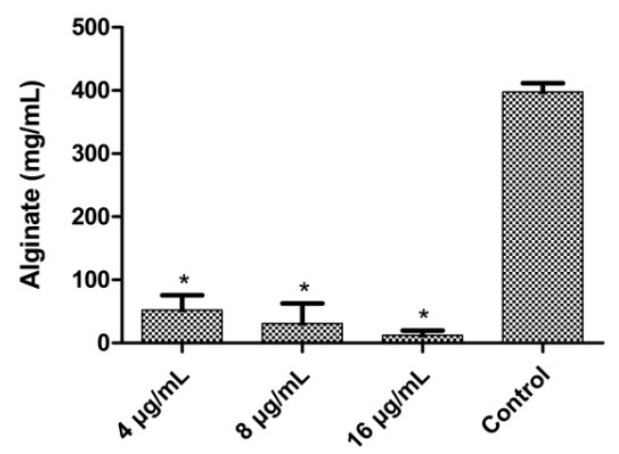

Fig. 2 d. The production of alginate. *Significance, $P<0.01$.

\subsection{The biofilm formation}

The biofilm formation decreased with the increasing of the concentration of PHE (Fig. 3). The biofilm formation reduced $66.6 \%, 51.9 \%, 40.9 \%$ respectively at third day, and $67.8 \%, 67.6 \%, 2.4 \%$ reduction for fifth day, $42.1 \%$, $35.4 \%, 11.1 \%$ reduction for 7 th day, respectively. By comparison, when the concentration was $4 \mu \mathrm{g} / \mathrm{mL}$, significant difference was only found on the third day.

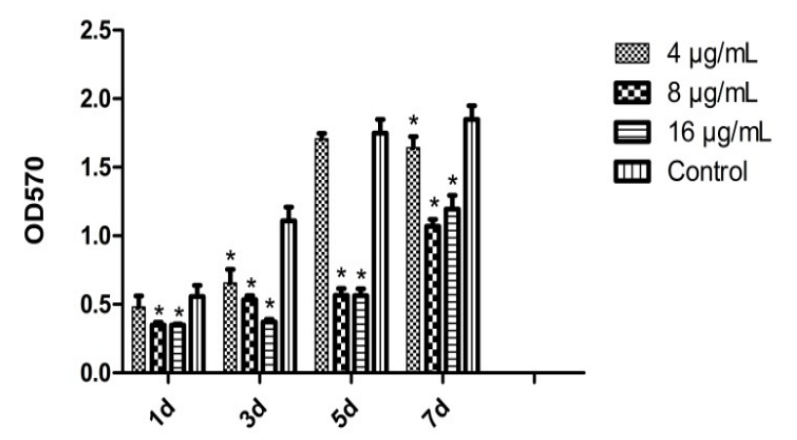

Fig. 3. The semi-quantitative of BF.

\section{Discussion}

Pseudomonas aeruginosa is widely distributed in nature, normal skin, intestinal and respiratory tract, and it is the main cause of respiratory tract infection, urinary tract infection, septicemia, osteomyelitis and skin infections [14]. QS enables the P. aeruginosa to synthesize and release a large number of extracellular virulence factors. Some researches have found that when QS system was blocked, an obvious reduction was detected in the secretion of virulence factors, biofilm formation, invasiveness of infected hosts [15].

Rhamnolipids is made up of two fatty acid molecules and rhamnose residues, which is controlled by Rhl system, mainly by rhlAB synthetic gene cluster expression [16]. It can decrease surface tension and biofilm development. The production of rhamnolipids reduced by $60.8 \%$ (B) and $9.7 \%$ (C) without affecting PAO1 growth. We could find a noticeable change when PHE concentration shifted to $8 \mu \mathrm{g} / \mathrm{mL}$ (B), and group B had a stronger inhibitation than group $\mathrm{A}$. Other studies showed that 6-gingerol $(0.1$ to $100 \mathrm{mM})$ inhibited rhamnolipid production by $36-60 \%$ [17]. 
Pyocyanin formation is regulated by a complex synchrony of rhlR-rhlI and lasR-lasI whereby interferences in these systems led to the deficiency of pyocyanin formation. Our study found pyocyanin production has a steady decrease with the increasing concentrations of PHE. In this test, the release amount of pyocyanin was kept at a low level in the positive and control groups. The results showed that PHE has slight effect on the production of pyocyanin in PAO1.

LasI/LasR system regulates the genes responsible for las A (protease) [18]. In the presense of PHE, protease activity reduced by $38.7 \%$ (B) and $14.7 \%$ (C). In comparsion, 6-gingerol inhibited protease production by $21-43 \%$ at concentrations ranging from 0.1 to $100 \mathrm{mM}$ [17], and parthenolide inhibited protease activity (45\%) at $1 \mathrm{mM}[18]$.

Alginate is an anionic polysaccharide and it is a vital element of biofilm. Alginate can build stronger bacteria adhesion, promote $\mathrm{BF}$ structure to mature, maintain the BF structure and enhance resistance to antibiotics $[8,19]$. It is also can inhibite quinolone signalling when overproduced [20]. As a result, the inhibition of alginate was $86.4 \%$ at $4 \mu \mathrm{g} / \mathrm{mL}$, and almost complete inhibition at higher concentrations.

P. aeruginosa biofilm can effectively resist the attack of antibiotics and immune system. Under the interference of conventional drug, biological membrane is difficult to be cleared, so infections caused by bacterial biofilms often show intractable and persistent problems. Three days is the formative period of biofilm, and seven days for biofilm stabilization, the stable biofilm forms a netted three-dimensional structure with the bacteria of the package. Biofilm can absorb nutrients to arrange itself to produce toxins into the surrounding environment to play the role of the second layer protection. Biofilm formation of $P$. aeruginosa is co-controlled by several QS system. Under the interference of PHE, biofilm formation in PAO1 reduced at least by $40.9 \%$ in the first three days. Siraj Datta et al. showed that piper betle leaf extract inhibited biofilm formation (in the first day) by $7.81 \%$, $32.54 \%, 66.16 \%$ at $50 \mu \mathrm{g} / \mathrm{mL}, 100 \mu \mathrm{g} / \mathrm{mL}, 150 \mu \mathrm{g} / \mathrm{mL}$, respectively [10]. Reserpine decreased biofilm formation by $17.12 \%$ at $200 \mu \mathrm{g} / \mathrm{mL}$ [8]. 6-gingerol (0.1 to $100 \mathrm{mM})$ decreased biofilm formation by $19 \%-53 \%$ [17]. Pure parthenolide decreased biofilm formation by $56 \%$ at 1 $\mathrm{mM}$ [18]. Pistacia atlantica decreased biofilm formation by $39 \%$ at $0.25 \mathrm{mg} / \mathrm{mL}$ [21].

To sum up, PHE has a stronger effect on alginate, rhamnolipids and biofilm formation at a very low concentrations $(8 \mu \mathrm{g} / \mathrm{mL}, 4 \mu \mathrm{g} / \mathrm{mL})$. The growth curve of PAO1 in the presence of PHE $(8 \mu \mathrm{g} / \mathrm{mL}, 4 \mu \mathrm{g} / \mathrm{mL})$ showed no variation as compared to PHE free medium. Based on the experimental results and available literature, it could be assumed that PHE might have inhibited the QS-systems in $P$. aeruginosa, and inhibited QS-controlled virulence factors production. PHE may become a potential good candidate for quorum sensing inhibitors.

\section{Conclusions}

As a traditional Chinese medicine, Plantain Herb is used for several thousand years. Our results have demonstrated Plantain Herb extract as a potential QS inhibitor. We also verified that the extract inhibited pyocyanin, protease and rhamnolipids production in $P$. aeruginosa PAO1. Active compounds in this extract might offer a new insight towards discovering potential anti-pathogenic drugs to combat emerging multidrug resistant pathogens. However, few questions remained to be answered for future study, what exactly the active compounds are?

\section{References}

1. D.I. Andersson. Persistence of antibiotic resistant bacteria. Curr Opin Microbiol. 6, 452-6 (2003).

2. N.C. Reading, V. Sperandio. Quorum sensing: the many languages of bacteria. FEMS Microbiol Lett. 254, 4-11 (2006).

3. A. Deep, U. Chaudhary, V. Gupta. Quorum sensing and Bacterial Pathogenicity: From Molecules to Disease. J Lab Physicians. 3, 4-11 (2011).

4. B. Mellbye, M. Schuster. The Sociomicrobiology of Antivirulence Drug Resistance: a Proof of Concept. mBio. 2, 119 (2011).

5. J. Tremblay, A.P. Richardson, F. Lépine, E. Déziel. Self-produced extracellular stimuli modulate the Pseudomonas aeruginosa swarming motility behaviour. Environ Microbiol. 9, 2622-30 (2007).

6. G.M. Dunny, B.A.B. Leonard. Cell-cell communication in gram-positive bacteria. Annu Rev Microbiol. 51, 527-64 (2003).

7. M. Qi, A. Xiong, F. Geng, L. Yang, Z. Wang. $A$ novel strategy for target profiling analysis of bioactive phenylethanoid glycosides in Plantago medicinal plants using ultra-performance liquid chromatography coupled with tandem quadrupole mass spectrometry. J SEP SCI. 35, 1470-8 (2012).

8. D. Parai, M. Banerjee, P. Dey, A. Chakraborty, E. Islam, S.K. Mukherjee. Effect of reserpine on Pseudomonas aeruginosa quorum sensing mediated virulence factors and biofilm formation. Biofouling. 1-15 (2018).

9. T. Rasamiravaka, O. M. Vandeputte, L .Pottier, et al. Pseudomonas aeruginosa Biofilm Formation and Persistence, along with the Production of Quorum Sensing-Dependent Virulence Factors, Are Disrupted by a Triterpenoid Coumarate Ester Isolated from Dalbergia trichocarpa, a Tropical Legume. Plos One. 10 (2015).

10. S. Datta, D. Jana, T.R. Maity, A. Samanta, R. Banerjee. Piper betle leaf extract affects the quorum sensing and hence virulence of Pseudomonas aeruginosa PAO1. 3 Biotech. 6, 18 (2016).

11. F.M. Husain, I. Ahmad, A.S. Althubiani, H.H. Abulreesh, I.M. Alhazza, F. Aqil. Leaf Extracts of Mangifera indicaL. Inhibit Quorum Sensing - 
Regulated Production of Virulence Factors and Biofilm in Test Bacteria. Front Microbiol. 8, 727 (2017).

12. Y. Hiroshi, K. Tetsufumi, K. Harumi, K. Takeshi. Interaction between Biofilms Formed by Pseudomonas aeruginosa and Clarithromycin. Antimicrob Agents CH. 37, 1749-55 (1993).

13. A. Busetti, G. Shaw, J. Megaw, et al. Marine-derived quorum-sensing inhibitory activities enhance the antibacterial efficacy of tobramycin against Pseudomonas aeruginosa. Mar Drugs. 13, 1-28 (2014).

14. H.K. Doshi, K. Chua, F. Kagda, P.A. Tambyah. Multi drug resistant pseudomonas infection in open fractures post definitive fixation leading to limb loss. $A$ report of three cases. International Journal of Case Reports \& Images 2, 1-6 (2011).

15. J. Lee, L. Zhang. The hierarchy quorum sensing network in Pseudomonas aeruginosa. Protein Cell. 6, 26-41 (2015).

16. T. Bjarnsholt, P Ø. Jensen, M. Burmølle, et al. Pseudomonas aeruginosa tolerance to tobramycin, hydrogen peroxide and polymorphonuclear leukocytes is quorum-sensing dependent.
Microbiology. 151, 373-383 (2005).

17. H.S. Kim, S.H. Lee, Y. Byun, H.D. Park. 6-Gingerol reduces Pseudomonas aeruginosa biofilm formation and virulence via quorum sensing inhibition. Sci Rep. 5, 8656 (2015).

18. M. Kalia, V.K. Yadav, P.K. Singh, D. Sharma, S.S. Narvi, V. Agarwal. Exploring the impact of parthenolide as anti-quorum sensing and anti-biofilm agent against Pseudomonas aeruginosa. Life Sci. 199, 96-103 (2018).

19. A.P. Stapper, G. Narasimhan, D.E. Ohman, et al. Alginate production affects Pseudomonas aeruginosa biofilm development and architecture, but is not essential for biofilm formation. J Med Microbiol. 53, 679-90 (2004).

20. J. Yang, M. Toyofuku, R. Sakai, N. Nomura. Influence of the alginate production on cell-to-cell communication in Pseudomonas aeruginosa PAO1. Environ Microbiol Rep. 9, 239-49 (2017).

21. H. Kordbacheh, F. Eftekhar, S.N. Ebrahimi. Anti-quorum sensing activity of Pistacia atlantica against Pseudomonas aeruginosa PAO1 and identification of its bioactive compounds. Microb Pathog. 110, 390-8 (2017). 